\title{
Lebensbilder aus Limbach-Oberfrohna
}

\author{
Christian Kirchner, Jürgen Lohr, Walter Wenzel, Ursula Ziemert
}

\section{Georg I. von Schönberg (1524-1588)}

Georg von Schönberg ${ }^{1}$, Sohn des ersten Schönberger Lehnsträgers auf Limbach, wurde am Gründonnerstag 1524 geboren. Er schrieb sich 1542 in der Universität Wittenberg ein und trat später in den Dienst des Kurfürsten Johann Friedrich von Sachsen. Das Rittergut Limbach erbte er 1546, wobei die offizielle Belehnung erst im Jahre 1552 erfolgte. Zu diesem Zeitpunkt schien er schon über ein stattliches Vermögen verfügt zu haben, was Pfandscheine an den kurfürstlichen Hof zeigen. $\mathrm{Zu}$ seinem Limbacher Erbe erhielt er bei dem Aussterben einer anderen Schönberger Nebenlinie 1576 das Schloss Sachsenburg mit Zubehör zugesprochen.

Wichtig für Limbach und das Rittergut war er aber vor allem durch seine Umgestaltung des Dorfkernes. So kaufte er zuerst 1552 das alte Schenkgut und ließ dieses 1572 neu erbauen. Für die Lagerung des Bieres ließ er den Kellerberg unterhöhlen und die namensgebenden Keller einrichten. Weiterhin ließ er um 1570 das Herrenhaus in massiver Bauweise errichten. Ein weiterer Bestandteil des damaligen Baugeschehens war die Neuerrichtung der Fronfeste. Der Kirche vermacht er einen steinernen Altar und gibt eine Spende an den Pfarrer sowie den

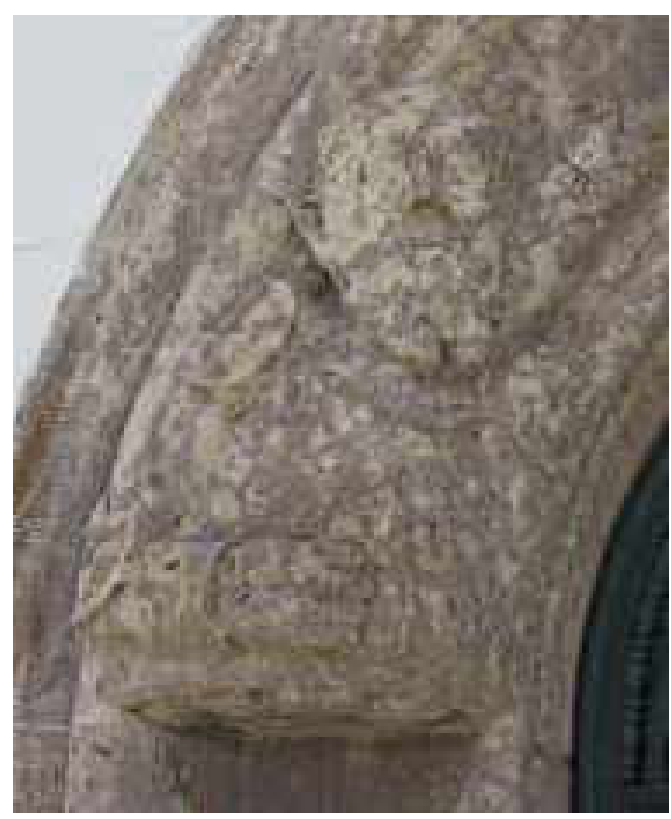

Schulmeister. Bis heute überdauert hat auch der Knauteich, welchen er durch Errichtung des Dammes anstauen ließ. Seine hiesigen Besitz vergrößert er 1585 durch Ankauf des Rittergutes Mittelfrohna. Georg I. von Schönberg verstarb am 5. Januar 1588 zu Sachsenburg und wurde am 16. Januar 1588 in der Kirche zu Limbach beigesetzt.

\section{Helena Dorothea von Schönberg (1729-1799)}

Die bedeutendste Frau in der Limbacher Geschichte wurde am 22. November 1729 zu Schweikershain als Tochter des Oberstleutnant Johann Joachim von Wallwitz und dessen Gemahlin Johanna Sophia von Bünau geboren. Ihre Kindheit verbrachte sie wohl auf dem elterlichen Gut und erhielt dort sicherlich die Ausbildung durch einen Hauslehrer. Im Alter von 16 Jahren heiratete sie am 27. September $1746 \mathrm{zu}$ Limbach den Oberwachtmeister des kurprinzlichen Kürassierregiments Georg Anton von Schönberg. Mit ihm zusammen begann sie, die wirtschaftliche Kraft des Dorfes zu forcieren. Zunächst wurde 1747 eine eigene Ziegelei und 1750 eine Klöppelschule im Rittergut eingerichtet; 1751 begann man mit der Nutzung des Serpentinsteinbruchs am Hohen Hain. 1750

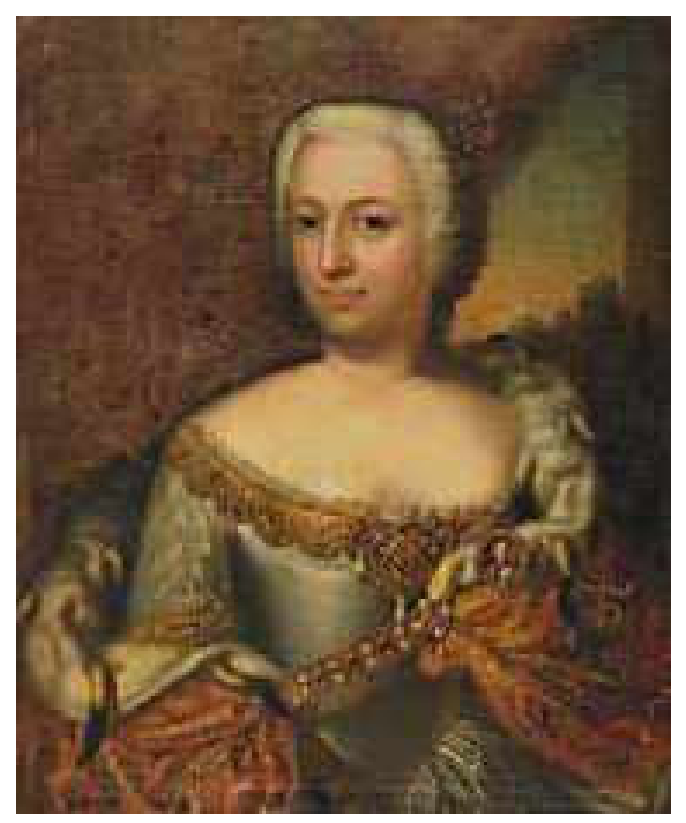

1 Die Beiträge zu Georg von Schönberg, Helena Dorothea von Schönberg, Johann Esche, Friedrich Ludwig Leuschner und Hans Möckel stammen von Christian Kirchner. Der Beitrag zu Johann Esche wurde von Matthias Donath unter Verwendung neuer Forschungsergebnisse von Dietrich Esche überarbeitet.

Herrenhaus Limbach, Sitznischenportal, vermutlich Bildnis Georg von Schönbergs

Foto: Matthias Donath
Helena Dorothea von Schönberg, Gemälde, um 1780

Foto: Dietmar Träupmann 
2 Der Beitrag zu Anna Esche stammt von Ursula Ziemert.

Johann Esche, Gemälde um 1750, Dauerleingabe aus Privatbesitz im Esche-Museum Limbach-Oberfrohna Foto: Dietmar Träupmann wurde mit dem Bau der ersten planmäßigen Wirkersiedlung Deutschlands auf dem Helenenberg begonnen. Nach der ersten Besiedlung verstarb am 25. Juli 1755 ihr Ehegatte und es kam zu Erbstreitigkeiten mit dessen Bruder Johann Dietrich. Außerdem entstanden durch den Siebenjährigen Krieg ungeheure Kosten, welche den wirtschaftlichen Aufschwung etwas bremsten. Noch während dieser Zeit wurde 1757 das neue Brauhaus (heute Hotel „Lay-Haus“) fertiggestellt.

Sie selbst konnte die Erbstreitigkeiten zu ihren Gunsten klären und wurde 1759 unter der Vormundschaft ihrer Brüder und eines Schwagers mit dem Rittergut Limbach belehnt. Dieses brannte mit Ausnahme des Herrenhauses im Jahre 1769 nieder. Das weiterbetriebene Wachsen der Siedlung am Helenenberg und die Anlage am Dorotheenberg brachten dem Dorf einen weiteren Zuzug von Strumpfwirkern, sodass nach langwierigen Verhandlungen im Jahre 1785 eine eigene Innung gegründet werden konnte. Das florierende Dorf bekam durch die Ausdauer seiner Rittergutsherrin 1795 das Recht auf zwei Jahrmärkte. Nach einem langen Leben, in dem sie für Limbach Entscheidendes in die Wege leitete, verstarb die Rittergutsherrin Helena Dorothea von Schönberg am 29. März 1799. An der Seite ihres Gemahls wurde sie in der Limbacher Kirche neben dem Altar beerdigt.

Johann Esche (1682-1752)

Limbachs bekanntester Einwohner des 18. Jahrhunderts wurde am 3. Mai 1682 in Köthensdorf getauft. Sein Vater zog mit der Familie 1698 nach Limbach und kaufte am Markt eine Färberei. Da Köthensdorf sowie auch Mittelfrohna, wo sein Vater Johann Esche herstammte, zum Lehen des Rittergutes Limbach gehörten, war dieser Wechsel recht unproblematisch. Über die Jugendjahre Jo-

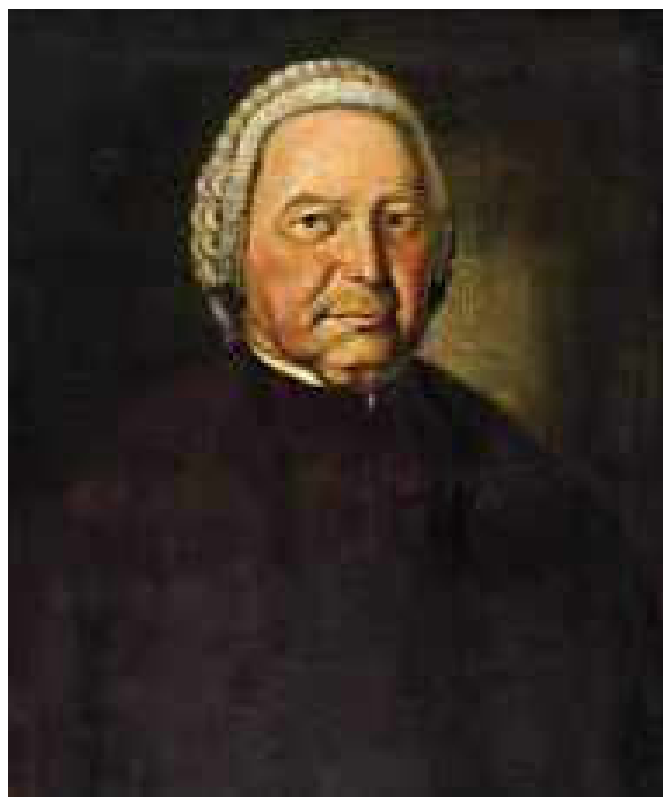

hann Esches ist nur sehr wenig bekannt. Forschungen von Dietrich Esche ergaben, dass er zunächst in der Färberei seines Vaters als Formenstecher tätig war. Dann wurde er Strumpfwirker. Wie die anderen Wirker in Limbach stellte er zunächst wollene Produkte her. Nach 1727 lernte er auf einer Reise nach Dresden einen Hugenotten kennen, der auf einem Wirkstuhl Seidenstrümpfe fertigte. Danach arbeitete Johann Esche in den folgenden Jahren an der Entwicklung eines Wirkstuhls, der Seide verarbeiten konnte. Esche wurde somit zum Begründer der Seidenwirkerei in Limbach und Umgebung, die allerdings zunächst nur auf wenige Meister beschränkt war. Johann Esche wurde über die Jahre ein angesehener Strumpf- und Seidenfabrikant sowie Handelsmann, der seine Waren auf der Messe in Leipzig mit großem Erfolg absetzte. Er kaufte ein Haus im später so genannten Esche-Viertel, in dem die Familie nach und nach mehrere Grundstücke erwarb und bebaute. Am 30. Januar 1752 verstarb Johann Esche als „erbangeseßener, alter wohlangesehener, werter Einwohner in Limbach“.

Johann Esche war der Stammvater der weit verzweigten Fabrikantenfamilie Esche. Sein Nachfahre Moritz Samuel Esche gründete 1843 in Limbach eine Strumpffabrik. Diese wurde 1871 nach Chemnitz verlegt und zur größten Strumpffabrik Deutschlands ausgebaut.

\section{Anna Esche (1824-1920)}

Am 23. Dezember 1824 erblickte in Chemnitz Anna Clara Clauß das Licht der Welt, die Tochter von Peter Otto Clauß, dem Gründer der Spinnerei in Flöha, und seiner Frau Henriette Sophie geb. Rahlenbeck. ${ }^{2}$ Am 18. Oktober 1844 heiratete sie in die in Limbach hoch geachtete Fabrikantenfamilie Esche ein. Ihr Mann Dr. Carl Julius Esche hatte Medizin studiert und zu-

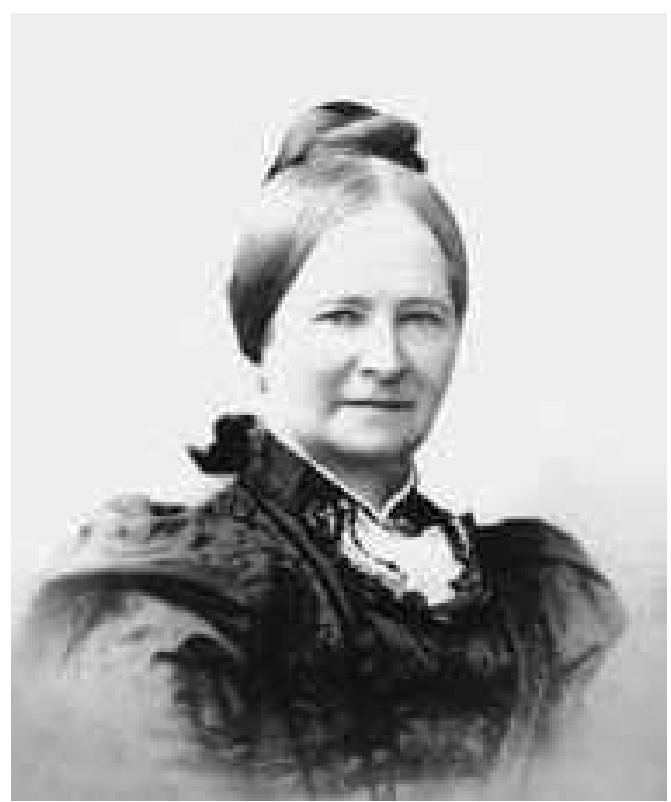


nächst als Arzt praktiziert. 1843 gab er seinen Beruf auf, um Teilhaber der Strumpffabrik Moritz Samuel Esche zu werden. Anna und Julius bekamen drei Kinder. Nach dem Tod ihres Mannes 1867 kümmerte sich Anna Esche bis ins hohe Alter um soziale Belange in Limbach.

Ihr erstes Projekt war 1882 die Gründung einer „Kinderbewahranstalt“, in der noch nicht schulpflichtige Kinder berufstätiger Frauen gegen ein geringes Entgelt ganztägig betreut wurden. Für den Neubau in der heutigen Südstraße, in dem schließlich 60 Kinder betreut werden konnten, stiftete sie im Jahr 189015.000 Mark und blieb auch weiterhin eine der tatkräftigsten Unterstützerinnen. An ihrem 80. Geburtstag im Dezember 1904 wurde die Einrichtung nach ihr benannt. Das „Anna-Esche-Heim“ bestand bis 1923. 1887 wurde Anna Esche Mitbegründerin und Vorsitzende des Albert-Zweigvereins. Dieser im Jahre 1867 in Dresden gegründete Frauenverein des Roten Kreuzes war nach König Albert von Sachsen benannt und widmete sich in Friedenszeiten karitativ der Armenpflege. Hauptaufgabe war die Betreuung sozial Benachteiligter und Kranker, die Verteilung kostenloser Lebensmittel und die Unterstützung von $\mathrm{Fa}$ milien, die fremde, oft uneheliche Kinder gegen geringes Entgelt aufzogen.

1896 wurde Anna Esche die erste Ehrenbürgerin Limbachs. Die Verleihung der Ehrenbürgerwürde wurde damit begründet, dass sie über 50 Jahre lang in Limbach durch „unermüdliche Wohltätigkeit die sichere Zuflucht der Armen und Bedrängten“ geworden sei. Gleichzeitig wurde zu ihren Ehren die Fachschulstraße in Anna-EscheStraße umbenannt. Auch das Königreich Sachsen verlieh Anna Esche hohe Auszeichnungen, so 1894 die silberne Carola-Medaille für ihre Verdienste im Albertverein und auf dem Gebiet der hilfreichen Nächstenliebe, 1896 der Sidonien-Orden und 1916 die Carola-Medaille in Gold. Anna Esche starb im Alter von 96 Jahren, bis zuletzt geistig rege und hoch geehrt, am 16. Februar 1920. Von ihr und ihren Kindern stammten verschiedene Stiftungen und Schenkungen, so Schenkungen für das Krankenhaus und die „Geschwister-Esche-Stiftung“, deren jährliche Zinserträge bedürftigen Männern und Frauen über 60 Jahre „zur Bezahlung von Wohnung und Feuerung“ "zugutekamen.

\section{Friedrich Ludwig Leuschner (1824-1889)}

Der erste bürgerliche Besitzer des Ritterguts Limbach wurde am 10. März 1824 in Gräfenhainichen geboren. 1850 erlangte er das Bürgerrecht zu Glauchau und gründete dort eine Fabrik. Im darauffolgenden Jahr vermählte er sich mit Henriette Elise Raum, der Tochter des Konsistorial- und Kanzleidirektors der Schönburgischen Gesamtkanzlei. Am 18. August 1862

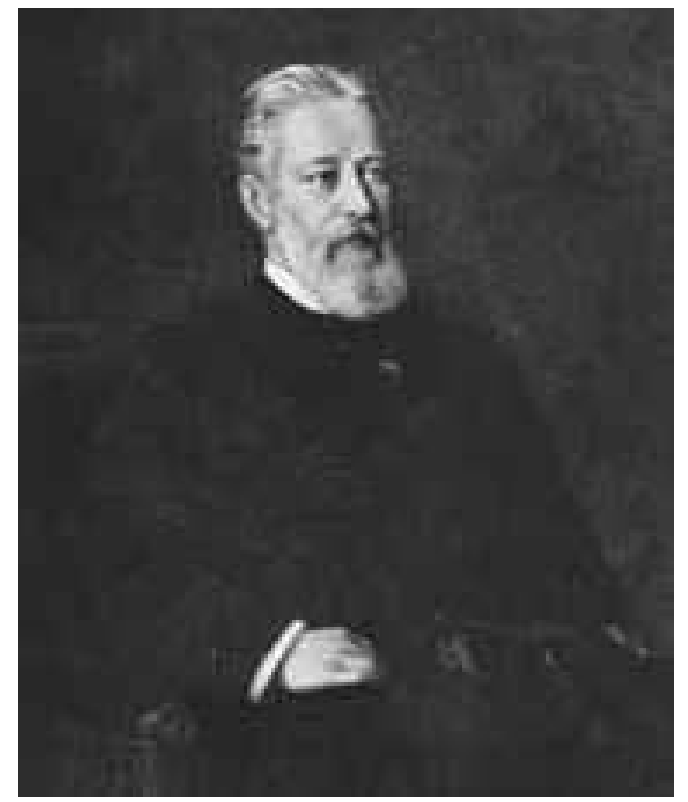

Friedrich Ludwig Leuschner, Gemälde, um 1889

๑) Stadtarchiv Limbach-Oberfrohna kaufte er das Rittergut Limbach von Freiherr Otto von Welck und begann gleich mit dem Schaffen von notwendigen Veränderungen. Er ordnete das Jahrmarktwesen neu und forcierte die Bebauung des Kellerbergs. Der neue Platz erhielt ihm zu Ehren die Bezeichnung Ludwigsplatz, da er das große Bassin stiftete, welche viele Jahre das Bild des Platzes prägte. Weiterhin zeigte er sich sehr rührig im sozialen Bereich, indem er sich um die Förderung des Schulwesens kümmerte und den Bau der Bürgerschule I vorantrieb. Als in den Jahren 1870/71 die deutschen Soldaten aus dem Feldzug gegen Frankreich verwundet nach Hause kamen, bot sich Rittergutsbesitzer Leuschner an, diese auf eigene Kosten und in seinem eigenen Haus persönlich unterzubringen und versorgen zu lassen. So kam es, dass 100 Verwundete in Limbach gesund gepflegt wurden. Zum Dank übereignete das Preußische Kriegsministerium ihm eine erbeutete französische Kanone, welche viele Jahre auf dem Gutshof prangte und für Salutschüsse angewandt wurde.

Zur Steigerung der Wirtschaftlichkeit der Gemeinde Limbach bedurfte es des Baues einer Bahnlinie. Da der direkte Anschluss an die Linie Leipzig-Chemnitz gescheitert war, wurde eine Zweiglinie ab Wittgensdorf eingerichtet, für welche eine Enteignung vieler Hektar Land aus dem Besitz des Rittergutes notwendig war. Auch politisch engagierte sich Leuschner für die hiesige Gemeinde und vertrat von 1873 bis 1878 den Wahlkreis in der II. Kammer des sächsischen Landtags. Nachdem er eine Petition zur Erteilung einer Städteordnung und damit der Stadtrechtsverleihung von Limbach eingereicht hatte, fiel er durch einen Unfall monatelang aus und legte sein Mandat nieder. Das Rittergut Limbach übergab er 1883 seiner ältesten Tochter Magdalene, welche mit Carl Lingelbach verheiratet war, 
3 Der Beitrag zu Heinrich Mauersberger stammt von Jürgen Lohr. und kaufte sich das Rittergut Dittersdorf in der Sächsischen Schweiz, ehe er am 29. Dezember 1889 in seinem Elternhaus zu Glauchau verstarb und dort in der Familiengruft beigesetzt wurde.

\section{Hans Möckel (1908-1942)}

Der Limbacher Architekt war über viele Jahrzehnte in Vergessenheit geraten, da seine Schaffenszeit in die Zeit des Dritten Reiches fiel. Hans Kurt Möckel wurde als Sohn des Kaufmanns Hans Otto Möckel am 28. Februar 1908 in der Helenenstraße 50 geboren. Er besuchte von 1914 bis 1922 die Bürgerschule I. Daran schloss sich eine Ausbildung als Maurer und Bautechniker bei der Firma des Baumeisters Curt Sussig an, welche er 1925 mit sehr guten Zeugnissen beendet. Er besuchte darauf bis 1927 die Sächsische Staatsbauschule für Hochbau in Chemnitz, wo er u. a. von Dr.-Ing. Hermann Heuss, dem Bruder des späteren Bundespräsidenten Theodor Heuss, unterrichtet wurde. Erste berufliche Erfahrungen als Hochbautechniker sammelte er 1927 bis 1929 bei Architekt Dr.-Ing. Wilhelm Sievers, wo er „eine große Anzahl Villen, Fabriken, Wohnhaus-Projekte, Wettbewerbe für Schulen, Ortskrankenkassen usw." mit entworfen und die Entwicklung überwacht hat. Zu weiteren Studien schrieb er sich an der Akademie für bildende Künste Dresden ein, um von 1929 bis 1933 die Meisterschule von Prof. Dr. Wilhelm Kreis zu besuchen. Diese schloss er mit mehreren Auszeichnungen und Ehrenzeugnissen ab. Neben diesem Studium war er aber auch praktisch tätig und überwachte u. a. die Neugestaltung der Lehrerwohnung in Pleißa, nahm an zahlreichen Wettbewerben teil und errang im Abschlussjahr den „Rompreis“,

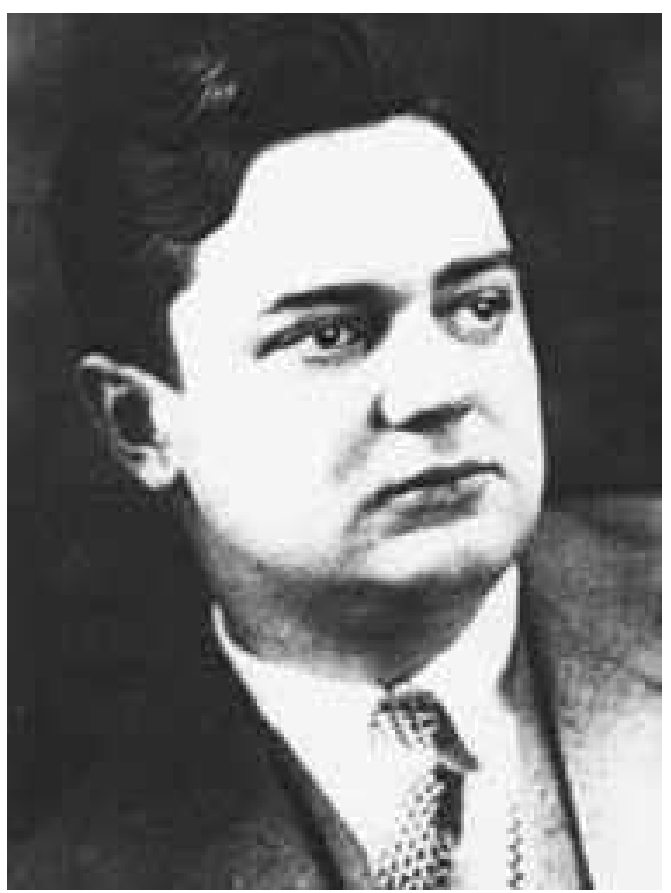

welcher ihm einen einjährigen freien Studienaufenthalt in Rom ermöglichte. Da er mit vielen weiteren Projekten beschäftigt war, nahm er diese Chance allerdings nur offiziell wahr und arbeitete in Limbach weiter an seinen Projekten. Das wichtigste hiervon war die Umgestaltung des Limbacher Stadtzentrums. Bereits 1933 hatte er die Ausschreibung gewonnen, welche den Umbau des Rittergutes zum Verwaltungszentrum vorsah. Weiterhin wurde der alte Kirchgarten umgestaltet und die Baubrache der ehemaligen Rittergutsschäferei zum Rathausplatz eingeplant. Seinen Entwürfen folgend entstanden die Bogengänge, der Weg zwischen Kirche und Rittergut, die großen Fenster in Standesamt und Stadtkasse sowie weitere Details inner- und außerhalb des Gebäudes. Diese Umgestaltung war für ihn eine berufliche Qualifikation, welche ihm viele weitere Projekte in der näheren Umgebung einbrachte, auch ohne dass er sich mit den politischen Kreisen seiner Zeit abgab oder in die Partei eingetreten wäre. Seine Achtung in der Bürgerschaft errang er aber auch durch seinen Einsatz für das Stadtparkfest, dessen Organisation er über mehrere Jahre unterstützte. Bis heute sind die Galapagos-Insel oder die Bäreninsel in Erinnerung, welche er in den Jahren 1935 und 1936 auf der Insel des Dreierlenteiches (heute im Teil des Tierparks) errichtete. Ein weiterer beruflicher Aufstieg wurde durch den Kriegsbeginn jäh unterbrochen, da Hans Möckel zur Wehrmacht eingezogen wurde. Bei einem Flug über dem Berg Salatin in der Slowakei stürzte er $1942 \mathrm{ab}$ und erlag nach der Notlandung seinen schweren Verletzungen.

\section{Heinrich Mauersberger (1909-1982)}

Heinrich Mauersberger ${ }^{3}$ verhalf als Erfinder, Wegbereiter, Maschinenbauer und textiler Entwickler seinem neuen Stoffherstellungs-Verfahren zur internationalen Anerkennung. Er schuf die wesentlichen Grundlagen für die Serienfertigung der Nähwirkmaschinen. Unter dem geschützten Warenzeichen MALIMO ${ }^{\circledR}$ (Mauersberger Limbach-Oberfrohna) wurden sowohl die Maschinen als auch die darauf hergestellten Erzeugnisse national und international erfolgreich vermarktet. Mit dem Maschinenhersteller VEB Nähwirkmaschinenbau Karl-Marx-Stadt und dem weltweit größten Nähwirkbetrieb VEB Malitex Hohenstein-Ernstthal war diese Region das Zentrum der Nähwirktechnik MALIMO.

Am 11. Februar 1909 wurde Heinrich Mauersberger in Neukirchen bei Crimmitschau geboren. Sein Berufswunsch war Maschinenbauer oder Elektriker. Sein Vater schickte ihn aber von 1929 bis 1931 in die Lehre als Färber in eine Tuchfabrik. Nach erfolgreichem Lehrabschluss folgten der Besuch der Webschule in 
Crimmitschau und der Akademie für Technik in Chemnitz mit dem Abschluss als Färbereitechniker. Am 4. Februar 1933 heiratete er in Crimmitschau Elsbeth Winterling, die Tochter eines Schlossermeisters. Im gleichen Jahr ging er auf „Wanderschaft“ und arbeitet jeweils befristet in mehreren Textilfabriken Sachsens. 1934 erhielt er eine feste Anstellung als Chemiker/Colorist bei der damals weltbekannten Limbacher Handschuhfabrik C. A. Kühnert A.G. im Werk Göppersdorf bei Burgstädt. Mit seiner Frau zog er in die dortige Fabrikwohnung. In der Firma hatte er für die Farbgebung und effektvolle Ausrüstung der Handschuhstoffe zu sorgen. Daraus entstanden seine ersten zum Patent angemeldeten Erfindungen, u. a. der Ätzdruck für Handschuhstoffe. Von 1941 bis 1946 war er Soldat und nach Kriegsende Sanitäter in amerikanischer Kriegsgefangenschaft.

In schwerer Nachkriegszeit konnte er nur noch kurze Zeit in der fast still stehenden Handschuhfabrik tätig sein. Durch Einfallsreichtum und handwerkliches Geschick wollte er die Not überwinden. Mit einer selbst gebauten HandFlachstrickmaschine erlernte er das Strickhandwerk, legte die Strick-Meisterprüfung an der Handwerkskammer ab, meldete ein Heimgewerbe an und verkaufte etwa zwei Jahre lang die selbst gefertigten Strickwaren an ein Chemnitzer Textilwarengeschäft.

1947 kam die entscheidende Idee für das Übernähen von Fadenlagen beim Beobachten, wie seine Frau schadhafte Stellen in Geweben mit der Nähmaschine ausbesserte, also „übernähte“. Es folgte in seiner Wohnung der Bau einfacher Modelle für eine neuartige Flächenbildungstechnik, die nach seinen Berechnungen eine Stoffproduktion von $60 \mathrm{~m} / \mathrm{h}$ leisten könnte. Demgegenüber lieferte eine Webmaschine damals nur 4 bis $5 \mathrm{~m} / \mathrm{h}$ ! 1948 stellte Mauersberger seine Erfindung bei der ostdeutschen Wirtschaftskommission und bei der Mitte des gleichen Jahres gegründeten VVB Textima vor. Eine Fachkommission der VVB Textima hielt die Erfindung für wenig bedeutend, erteilte aber immerhin Mauersberger 1949 den Auftrag, als „Spezialkonstrukteur für Entwicklung“ im VEB Sicht- und Zerlegewerk Limbach ein Funktionsmuster zu bauen.

Das Patent mit dem Titel „Verfahren zur Herstellung von Kettelwebstoff“" stellte Mauersberger am 31. Januar 1949 fertig und hinterlegte es am 2. Februar 1949 im Patentamt. Am 3. Februar 1949 wurde das Patent WP8194 unter dem neuen Titel „Verfahren zur Herstellung von Kettenstichware" vom Amt für Erfindungs- und Patentwesen in der Gruppe der „Vielnadelnähmaschinen“ erteilt. Erst später erfolgte national und international die Eingliederung des Patentes in die Gruppe der „Kettenwirkmaschinen“. Nach mehreren vorläufigen Begriffen wie „Kettelweben“, „Kettenstechen“ oder „Malimieren“ prägte Mauersberger

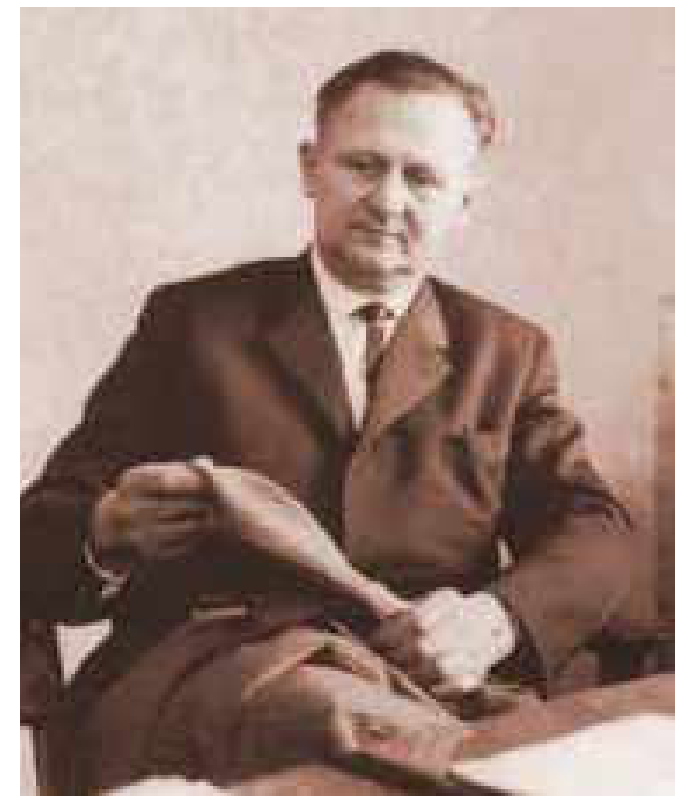

Heinrich Mauersberger, um 1965

$\odot$ Esche-Museum Limbach-

Oberfrohna mit Fachkollegen und Patentingenieuren etwa 1953 das neue Wort „Nähwirken“, dass sich schnell weltweit durchsetzte.

Vorher musste Mauersberger aber noch lange und zäh um die Anerkennung und die praktische Umsetzung seiner neuartigen Nähwirktechnik kämpfen. Er wehrte sich gegen Hohn, Spott und totale Ablehnung. Das Misstrauen war besonders groß, da Mauersberger kein ausgebildeter Konstrukteur war und als Autodidakt über Funktionsmodelle seine Technik entwickelt hatte. Mit vielen Vorträgen und weiteren in Eigenbau technisch verbesserten Modellen sowie den darauf hergestellten Nähgewirken suchte er Anerkennung und Unterstützung. Und er meldete weitere Patente zu seiner Nähwirktechnik an.

Etwa Anfang 1952 fand Mauersberger doch noch die „von oben“ notwendige wirtschaftspolitische Zustimmung für seine Nähwirktechnik, denn die VVB Textilmaschinenbau erhielt den Auftrag, mit Mauersberger den Bau eines ersten Fertigungsmusters in der Nennbreite 2400 Millimeter zu realisieren. Konstruktion und Teilefertigung erfolgten im VEB Konstruktion und Entwicklung KETEX Chemnitz, VEB Wirkmaschinenbau Limbach-Oberfrohna und VEB Spezialnähmaschinenbau Limbach-Oberfrohna. Im Werk Kändler des VEB Spul- und Schärmaschinenbau Burgstädt erfolgte die Montage. Mauersberger war Konstrukteur, Teilefertiger, Nadelmacher und Monteur. Als „verrückter Erfinder“ wurde er anfangs belächelt, aber durch seine Tatkraft, Ausdauer und Kollegialität fand er bald echte Weggefährten für die Nähwirktechnik.

Auf der Leipziger Frühjahrsmesse 1953 wurde die „Faserpelz-Kettenstich-Maschine“ (die erste MALIWATT) mit großem Erfolg ausgestellt. Der VEB Wirkmaschinenbau Limbach-Oberfrohna erhielt daraufhin den Auftrag zum Bau von drei Nullserien-Maschinen. 
In der Filzfabrik Oschatz war Mauersberger 1954 bei der Industrieerprobung seiner ersten Maschine dabei, um noch bestehende Mängel $\mathrm{zu}$ erkennen und abzustellen. Am 16. April 1954 konnte die arbeitsbereite Maschine die Produktion beginnen. Das Erzeugnis, die neue „Steppwatte“, fand aber keinen Absatz! Mauersberger suchte und fand schließlich selbst einen Abnehmer, den VEB Wattana Lichtenstein bei Zwickau. Der Durchbruch war geschafft und 1954 erhielt Mauersberger den DDR-Nationalpreis für Wissenschaft und Technik.

1955 bezog Mauersberger mit Unterstützung der Stadt Limbach-Oberfrohna das neuerbaute Siedlungshaus am Oesterholz. In den Jahren ab 1953 arbeitete Mauersberger in Karl-MarxStadt im VEB KETEX, im VEB Wirkmaschinenbau und dann im Januar 1957 im neu gegründeten Institut für Textilmaschinen als Abteilungsleiter an der technischen und technologischen Weiterentwicklung der Nähwirktechnik.

1956 übernahm der VEB Tüllmaschinenbau (später umbenannt in VEB Nähwirkmaschinenbau) in Karl-Marx-Stadt die Konstruktion, Weiterentwicklung, Fertigung und Montage von Nähwirkmaschinen MALIMO in Serienproduktion. Die Konstruktion des neuen Typs MALIMO wurde 1957 abgeschlossen. Die 1958 gebauten Maschinen MALIMO 500 erhielten ab 1959 ihren Industrieeinsatz im Werk Cranzahl des VEB Wirkwaren Cunnersdorf als sogenannte „Handtuch-Maschinen“. Etwa 1957 fertigte Mauersberger parallel dazu in seiner Freizeit und in seiner Limbacher Wohnung das sogenannte UrModell des Typs MALIMO, auf dem er bis etwa 1959 über 400 Muster der „Fadenlagen-Nähgewirke“ entwickelte und herstellte.

1961 wurden zur Leipziger Frühjahrsmesse die ersten Nähwirkmaschinen der Typen MALIMO und MALIPOL in der größeren Nennbreite 1.600 Millimeter vorgestellt. Im gleichen Jahr erprobte der VEB Baumwollwebereien Hohenstein-Ernstthal diese MALIMO-Maschinen und wurde bald der erste größere DDR-Hersteller von Nähwirkerzeugnissen. 1964 wurde der Betrieb in VEB Malitex umbenannt und die Produktion vergrößert, es entstanden Nähwirkereien in den Werken Hohenstein-Ernstthal, Kaufungen und Wolkenburg. Am 19. Januar 1962 wurde der Warenzeichenverband für Nähwirkmaschinen und Nähwirkerzeugnisse mit Sitz in Hohenstein-Ernstthal gegründet, Mauersberger war Mitglied des Vorstandes. Dem Verband gelang es relativ schnell, in der DDR die Nähwirkbetriebe, den Nähwirkmaschinenbau sowie Forschungs- und Exportbetriebe für Nähwirktechnik als Mitglieder unter dem gemeinsamen Warenzeichen MALIMO $^{\circledR} \mathrm{zu}$ vereinen.

Mauersberger war 1962 beteiligt an der Vergabe der Lizenz zur Fertigung von Nähwirkmaschinen an die USA-Firma Crompton and Knowles. Am
7. Oktober 1963 wurde Mauersberger als „Held der Arbeit“ ausgezeichnet und am 14. Oktober zum Ehrenbürger der Stadt Limbach-Oberfrohna ernannt.

In den 1960er Jahren begann der internationale Durchbruch für die Nähwirktechnik. Die zweite Generation der MALIMO-Maschinen „Modell 14010“ wurde in viele Länder der Erde exportiert. Viele neue Erzeugnisse in der Bekleidung, vor allem aber für Heim und Haushalt sowie für technische Zwecke, kamen auf den Weltmarkt. Auf vielen Auslandsreisen informierte Mauersberger selbst über seine Nähwirktechnik, dabei konnte er auch dank seiner guten englischen und französischen Sprachkenntnisse viele Exportgeschäfte anbahnen. In der DDR entstanden weitere Nähwirkbetriebe und MALIMO-Erzeugnisse waren nahezu in jedem DDR-Haushalt zu finden.

Weil Mauersberger Kritik an den DDR-Wirtschaftsverhältnissen äußerte und sich von Mitarbeitern der Staatssicherheit nicht reinreden ließ, wurde er zunehmend „politisch unbequem“. Aus gesundheitlichen Gründen kündigte 1966 Mauersberger seinen Arbeitsvertrag im Institut für Textilmaschinen. Er wurde sogar in die Psychiatrie nach Waldheim eingewiesen! Nach etwa einem halben Jahr wurde er mit der Diagnose „ohne Befund“ entlassen. Mit 58 Jahren siedelte er 1967 mit seiner Frau fluchtartig nach Bestensee bei Berlin um. Zwei Jahre lebte er von seinen persönlichen Rücklagen ohne jegliches Einkommen. Die noch offenen USA-Lizenzeinnahmen blieben aus, stattdessen erhielt er eine bescheidene Lizenz-Anerkennungs-Prämie als Vergütung. Inzwischen erfuhren westdeutsche Textil-Berufskollegen vom Schicksal Mauersbergers und starteten in ihrer Fachzeitschrift einen Solidaritätsaufruf: „DDR-Erfinder Malimo nagt am Hungertuch“. Das war für die DDR-Behörden Anlass genug, Mauersberger schließlich eine Ehrenpension des Ministerrates zu gewähren.

1972 starb seine Frau Elsbeth, die ihm stets eine wichtige Begleiterin und moralische Stütze war. Im gleichen Jahr wurde Mauersberger anlässlich des zehnjährigen Bestehens des Warenzeichenverbandes MALIMO $^{\circledR}$ zum Ehrenvorsitzenden gewählt. Zur Internationalen Textilmaschinenausstellung ITMA erhält er die Urkunde und Medaille der Stadt St. Louis (Frankreich).

1976 heiratete Mauersberger Lisa Messerschmidt, eine gelernte Textilfacharbeiterin aus Trusetal/Thüringen. Seine zweite Frau richtete ihn wieder auf und begleitete ihn in seinem doch noch recht aktiven Ruhestand in Bestensee. 1979 wurde ihm als zweitem Deutschen die Ehrenmitgliedschaft des Textilinstituts Manchester (England) verliehen, eine sehr hohe und sehr seltene Auszeichnung. Am 16. Februar 1982 verstarb Heinrich Mauersberger und wurde in Bestensee 
auf dem Nordfriedhof beigesetzt. Er bleibt als stets bescheidener und hilfsbereiter Mann mit Witz und Humor in Erinnerung, der lebenslustig und freundlich zu jedermann war und durch den beharrlichen Einsatz für seine Nähwirktechnik weltweite Anerkennung fand. Mauersberger war selbst an über 80 Patentanmeldungen beteiligt, basierend auf seinen Ideen haben sich viele technisch-technologisch neuartige Lösungen durchgesetzt.

\section{Hans Walther (1921-2015)}

Am 9. Juli 2015 verstarb im 95. Lebensjahr Hans Walther, emeritierter Professor der Universität Leipzig, ein weit über Sachsen hinaus bekannter Namenforscher und Landeshistoriker. ${ }^{4}$ Geboren am 30. Januar 1921 in einer Lehrerfamilie in Oberfrohna, besuchte er die dortige Grundschule, legte in Chemnitz das Abitur ab und wurde danach zum Wehrdienst eingezogen. Nach Kriegsende arbeitete er eine zeitlang als Neulehrer, um dann, an die Universität Leipzig delegiert, das Studium in den Fächern Germanistik, Anglistik und Geschichte aufzunehmen. 1955 promovierte er mit der Dissertation „Die Orts- und Flurnamen des Kreises Rochlitz", die 1957 als Band 3 in der Reihe „Deutsch-Slawische Forschungen zur Namenkunde und Siedlungsgeschichte“ erschien.

1954 berief man ihn in die von dem Germanisten Theodor Frings und dem Slawisten Rudolf Fischer begründete „Leipziger namenkundliche Arbeitsgruppe“, in der er bis zu seiner Emeritierung im Jahre 1986 wirkte. Die Arbeitsgruppe, später ein selbständiger Wissenschaftsbereich der Sektion Theoretische und angewandte Sprachwissenschaft, stand in enger Verbindung mit der Sächsischen Akademie der Wissenschaften, als deren Mitglied Hans Walther im Rahmen der Historischen Kommission bis zu seinem Lebensende aktiv tätig war. Zusammen mit dem Slawisten Ernst Eichler erarbeitete er als Historiker und Germanist mehrere der 41 Bände der Reihe „Deutsch-Slawische Forschungen zur Namenkunde und Siedlungsgeschichte“ und betreute viele in dieser Reihe erschienene Monographien, von denen die meisten aus Dissertationen oder Habilitationen hervorgingen. Eine Synthese seiner langjährigen Bemühungen um die Zusammenführung von Germanistik, Slawistik und Landesgeschichte stellt seine 1968 angenommene und 1971 im Druck als Band 26 der genannten Reihe erschienene Habilitationsschrift „Namenkundliche Beiträge zur Siedlungsgeschichte des Saale- und Mittelelbegebietes bis zum Ende des 9. Jahrhunderts“ dar. Seine langjährigen Erfahrungen in der Forschung kamen auch seiner Lehrtätigkeit zugute, die er seit 1975 als Dozent und seit 1978 als

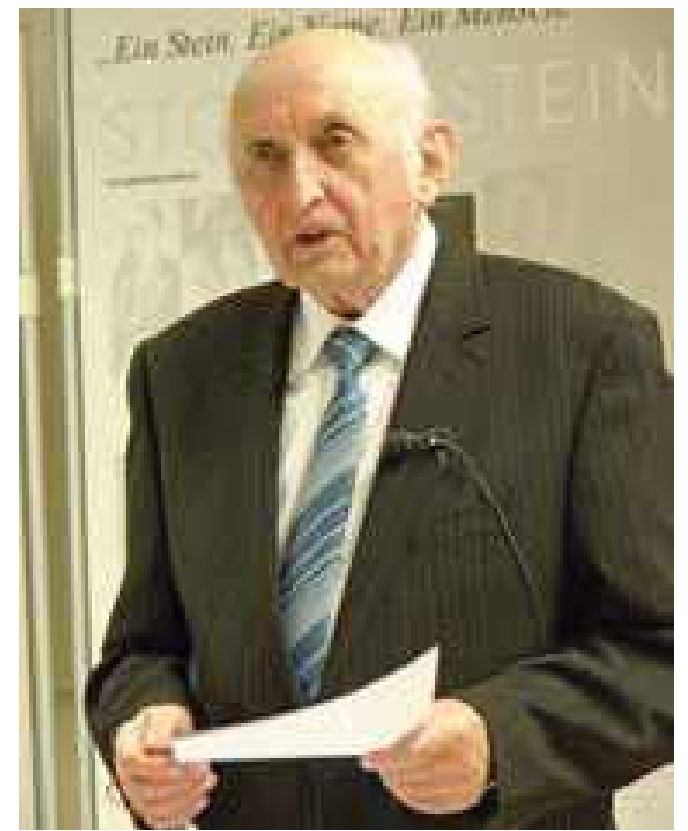

Hans Walther, 2010

Foto: Dieter Kremer

Professor bei der Heranbildung des wissenschaftlichen Nachwuchses und der Betreuung von Diplomanden und Doktoranden leistete. Gleichzeitig wirkte er an der Herausgabe und Redigierung mehrerer Zeitschriften sowie Reihen mit, so der „Namenkundlichen Informationen“, der „Deutsch-Slawischen Forschungen“, des „Jahrbuches für Regionalgeschichte“ sowie der deutsch-polnischen Zeitschrift „Onomastica Slavogermanica“. Darüber hinaus war er aktiv in verschiedenen wissenschaftlichen Vereinigungen tätig, so u. a. im Internationalen Komitee für Namenforschung (ICOS), in der Onomastischen Kommission beim Nationalkomitee der Slawisten der DDR, als Mitglied der Sächsischen Akademie der Wissenschaften in deren Gremien.

Auch nach seiner Emeritierung im Jahre 1986 war Hans Walther noch bis ins hohe Alter unermüdlich tätig. So verfasste er in der von Karl Czok 1989 edierten „Geschichte Sachsens“ die Kapitel II und III zur Landnahme und Stammesbildung der Sorben (um 600 bis 929), zur Markgrafschaft Meißen und zur Errichtung der deutschen Feudalherrschaft östlich der Saale mit Hilfe eines Burgwardsystems sowie zur Christianisierung (929-1156). 2001 gab er zusammen mit Ernst Eichler das dreibändige „Historische Ortsnamenbuch von Sachsen“ heraus, bearbeitet von ihm sowie Ernst Eichler, Volkmar Hellfritzsch und Erika Weber.

Die sächsische Geschichts- und Namenforschung verlor in Hans Walther einen ihrer herausragendsten Vertreter, das Limbacher Land in ihm einen seiner besten Söhne. Er wird allen seinen Freunden, Kollegen und Schülern als ein vorbildlicher Wissenschaftler und Lehrer, als liebenswürdiger und stets hilfsbereiter Mensch für immer in Erinnerung bleiben.
4 Dieser Nachruf stammt von dem Leipziger Namenforscher Prof. Dr. Walter Wenzel. 